\title{
Reducing the number of days taken for coronary artery bypass grafting for cardiology in-patients, within New Cross Hospital, Wolverhampton - a quality improvement project
}

\section{(D)Sam Hughes*, (i) Camilla Baker, (1D)Abhirami Gautham}

New Cross Hospital [Royal Wolverhampton Trust], Department of Cardiology, Wolverhampton, United Kingdom

\begin{abstract}
KEYWORDS: cardiology, coronary artery bypass grafting, quality improvement project, surgery. CITATION: Cardiol Croat. 2019;14(9-10):210. | https://doi.org/10.15836/ccar2019.210

*ADDRESS FOR CORRESPONDENCE: Sam Huges, Apartment 85, The Glasshouse, 3 Canal Square, Birmingham, B168FL, United Kingdom. / Phone: +447590629988 / E-mail: sam.hughes2@nhs.net

ORCID: Sam Hughes, https://orcid.org/0000-0002-3655-131X • Camilla Baker, https://orcid.org/0000-0002-1653-7684 Abhirami Gautham, https://orcid.org/0000-0001-9726-2805
\end{abstract}

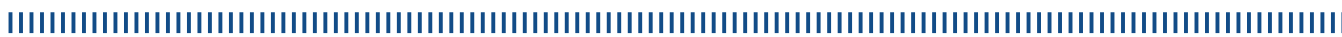

Background: Following an acute coronary event, the current process of referring a patient for Coronary Artery Bypass Grafting (CABG) at New Cross Hospital, Wolverhampton, has the potential to be improved. At present, patients can wait up to 5 days before being referred to cardiothoracics, often becoming more unwell during the delay. At times, patients become too unwell to undergo the planned surgery. Moreover, additional delays frequently arise due to difficulty tracking the referral.

Objective: To reduce the number of days between coronary angiogram to cardiothoracic review from 3 to 1 AND the number of days between coronary angiogram to CABG from 6 to 5 .

Method: The following 2 measures / PDSA cycles were implemented; - Cycle 1: Same-Day Referrals Upon reviewing the coronary angiogram, if the consultant cardiologist on-call deems the patient to be a 'clear surgical candidate', then a 'same day' referral to cardiothoracics for pre-assessment via the Teliologic system is made. - Cycle 2: 'Post-

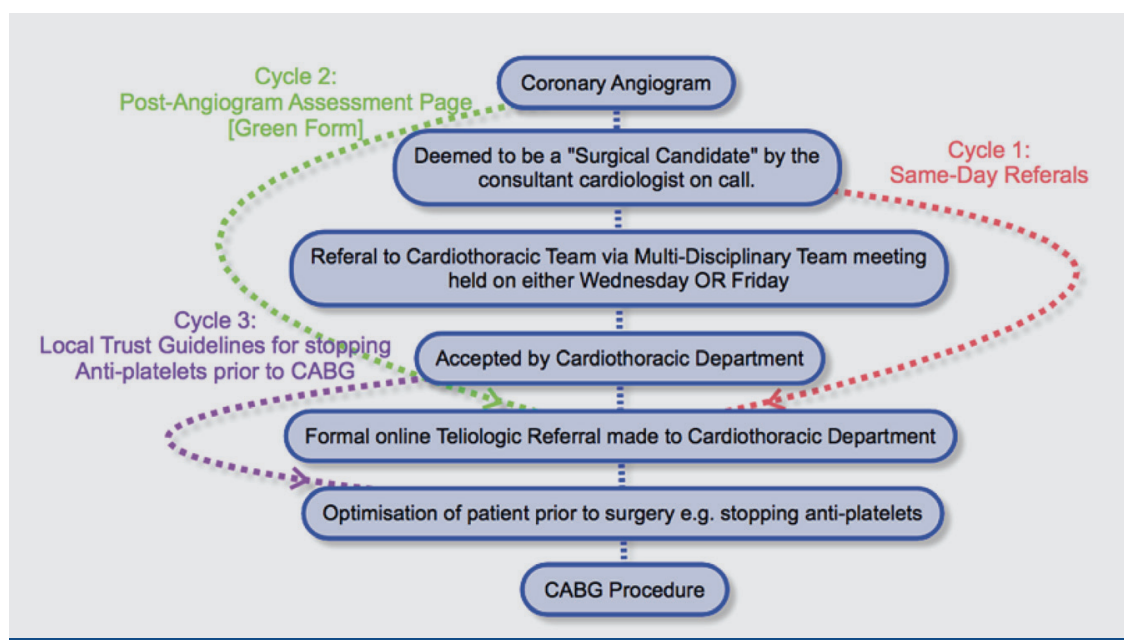

FIGURE 1. Flow chart outlining the current process of referring cardiology inpatients for coronary artery bypass grafting (CABG) procedure at New Cross Hospital, with each intervention labelled to highlight where in the chain the intended benefit will occur in order to increase overall efficiency of the process.
Angiogram' Assessment Page [Green Form] - Instead of utilizing numerous non-specific continuation sheets, a dedicated pro-forma is used to document the coronary angiogram results and to track the patient's subsequent plan (Figure 1).

Results: Coronary angiogram to cardiothoracic review reduced from 3 to 2 days, whilst from coronary angiogram to CABG reduced from 6 to 5 days. In addition, the average total length of stay has reduced by 3 days, improving patient outcomes and saving the hospital an average of $£ 1200$ per patient.

Discussion and Conclusion: At present, the decision on when anti-platelets [Ticagrelor in particular] should be stopped prior to surgery, varies from 2 days to 7 days, depending on both the patient's presentation \& the personal preference of each surgeon. ${ }^{1}$ This is the focus of Cycle 3, namely to implement local trust guidelines on stopping anti-platelets prior to CABG. Simple improvements in communication have significantly increased the efficiency of this referral process. For example, in cycle 2 we created a single, unified pro-forma which could be easily identified \& updated by each member of the team, improving consistency, continuity of care \& avoiding potential delays for surgery due to mis-placed documentation. In turn, this has reduced patient morbidity \& made significant cost savings.

LITERATURE IIIIIIIIIIIIIIIIIIIIIIIIIIIIIIIIIIIIIIIIIIIIIIIIIIIIIIIIIIIIIIIIIIIIIIIIIIIIIIIIIIIIIIIIIIIIIIIIIIII

1. Janssen PWA, Claassens DMF, Willemsen LM, Bergmeijer TO, Klein P, Ten Berg JM. Perioperative management of antiplatelet treatment in patients undergoing isolated coronary artery bypass grafting in Dutch cardiothoracic centres. Neth Heart J. 2017 Sep;25(9):482-489. https://doi.org/10.1007/s12471-017-1006-z $\square$ Cardiologia Croatica 2019;14(9-10):210. 